\title{
Advances and Applications in Stem Cell Biology
}

\author{
Shamoli Bhattacharyya
}

\begin{abstract}
Mesenchymal stem cells have shown great promise as the source of adult stem cells for regenerative medicine. Present research efforts are directed at isolating these cells from various sources, growing them in vitro and maintaining their pluripotency as well as capacity for self renewal. It is crucial to identify the regulatory molecules which directly or indirectly control the proliferative status or influence the niche microenvironment. The main challenge is to understand the basic biology of the stem cells and manipulate them for further therapeutic applications. Considering their malignant potential, stem cells may be a double edged sword. While the benefits of these cells need to be harnessed judiciously, a significant amount of research is required before embarking on widespread use of this tool for the benefit of humanity.
\end{abstract}

Keywords: Stem cells, Therapy, Differentiation, Applications.

How to cite this article: Bhattacharyya S. Advances and Applications in Stem Cell Biology. J Postgrad Med Edu Res 2012;46(2):75-80.

\section{Source of support: Nil}

Conflict of interest: None declared

\section{INTRODUCTION}

The goal of any health intervention is to maximize the therapeutic potential while minimizing the risk of adverse effects. Stem cell research has opened up new and exciting avenues in biological and biomedical sciences. Wide applications of stem cells and the development of cell-based therapies make it imperative to understand the basic biology of stem cells and to identify the factors governing cell dynamics. In recent years, regenerative medicine using stem cells offer great potential for tissue engineering and all based therapy. Tissue engineering approaches commonly involve seeding of cells at various stages of differentiation within scaffolds to fabricate new body tissues. ${ }^{1}$ In addition, in many tissues, they serve as a sort of internal repair system, dividing essentially without limit to replenish other cells as long as the person or animal is still alive. When a stem cell divides, each new cell has the potential either to remain a stem cell or become another type of cell with a more specialized function, such as a muscle cell, a red blood cell or a brain cell. It is important to translate the existing stem cell research platform for therapeutic applications that have predicable and adequate clinical effectiveness and safety. Apart from the host-donor cellular factors, the overall environment in which stem cells function must be considered for understanding the variable outcomes associated with stem cell based health interventions. The source of stem cells must also be carefully chosen based on functional and physical criteria that can lead to the desired outcome. ${ }^{2}$

\section{BASIC BIOLOGY OF STEM CELLS}

The human body is constantly regenerating blood cells, skin cells and some other tissues although it can not regenerate entire missing parts, like lizards or starfish. This has been possible due to the presence of a unique population of cells called the stem cells. In scientific terms, stems cells are the precursor cells with the ability to self renew, remain in an undifferentiated state and then terminally differentiate to a more specialized state in response to specific inductive signals. Stem cells have a long life span, relative quiescence, resistance to drugs and toxins through expression of multidrug resistance transporters, an active DNA repair capacity and resistance to apoptosis. ${ }^{3}$ On cell division, the stem cell can give rise to a daughter cell that is identical to itself (symmetric division) or a cell with limited developmental potential (asymmetric division). ${ }^{4}$ Asymmetric division is responsible for maintaining the stem cell identity and self renewal. This also leads to lineage specialization into a more differentiated cell type (Fig. 1). In many tissues, these act as an internal repair system, dividing to replenish other cells as long as the person or animal is alive. Nonstem cells, on the other hand are committed to a single lineage. Stem cells also show high plasticity, cross lineage barriers and adopt the expression profile and functional phenotypes of the cells that are typical of other tissues. This phenomenon is called transdifferentiation which describes the conversion to a cell of different type acquiring new cell fate, genetic repertoire and functions exactly of the transdifferentiated cell type. ${ }^{5}$ The concept that the identity of a somatic cell can be changed became a reality as lineage reprogramming was established in 2006. Yamanaka and Takahashi discovered how to 'reprogram' adult cells with specialized function (skin cells) in the laboratory, so that they behave like an embryonic stem cell. These cells, called induced pluripotent cells or iPS cells, were created by inducing the differentiated cells to express genes that are normally present in embryonic stem cells and that control the cell functions. ${ }^{6}$ This was possible through the achievement of a more primitive state and the successive differentiation to another cell type. The stem cells are generally classified according to their differentiation potential as shown in Table 1. 
Stem cells are classified as of embryonic origin (ESCs) or postnatal derivation (ASCs) based on the developmental stage from which they are obtained. ${ }^{7}$ The cells derived from various sources differ in potential to transform into cells of various lineages (Fig. 2).

Embryo-derived stem cells: Embryonic stem cells are derived from the inner cell mass of the blastocyst (a very early embryo). These have finite replicative lifespan after which they can no longer divide and are said to have reached a proliferative senescence. ${ }^{8}$

Fetal stem cells (FSC): FSCs are multipotent cells located in the fetal tissue and embryonic annexes. The only safe and relatively feasible source of FSC is fetal blood. ${ }^{9}$

Adult stem cells (ASC): Adult stem cells are partially committed stem cells localized in specific stromal niches. These are found in the majority of organs and tissues in adult organisms, and may help in long-term tissue maintenance and/or repair. ASCs can be obtained from bone marrow, ${ }^{10}$ muscle ${ }^{11}$ synovium, ${ }^{12}$ skin, ${ }^{13}$ deciduous teeth,${ }^{14}$ nerve tissue, ${ }^{15}$ etc.

Hematopoietic stem cells (HSCs) are well characterized cells of mesodermal origin deriving prevalently from BM. HSCs generate all mature blood cell types of the hematolymphatic system. Mesenchymal stem cells (MSCs) are generally restricted to forming only mesodermal specific cell types, such as adipocytes, osteoblasts, myocytes and chondrocytes, but several MSCs are able to differentiate in cells of the three embryonic germ layers. ${ }^{16}$ Recent studies show that cultured MSCs secrete various bioactive molecules which have got antiapoptotic, immunomodulatory, angiogenic, antiscarring and chemoattractant properties, providing a basis for their use as tools to create local regenerative environments in vivo. ${ }^{17}$

Umbilical cord stem cells: In the umbilical cord, we can find two types of SC sources, i.e. the umbilical cord epithelium (UCE), from the amniotic membrane epithelium and the umbilical cord blood (UCB) ${ }^{18}$ It has been

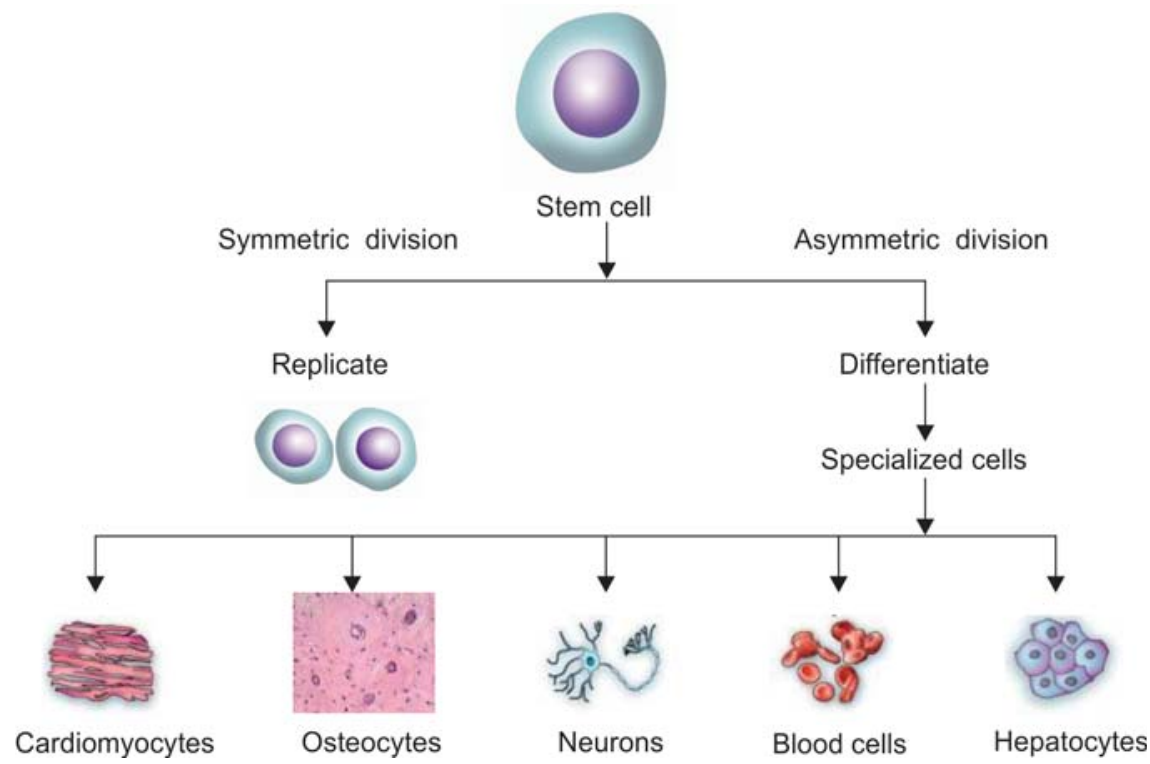

Fig. 1: When stem cell divides, each new cell has the potential either to remain a stem cell or become another type of cell with a more specialized function, like cardiac, red blood cell or neuron

\begin{tabular}{|c|c|c|c|}
\hline Sr no. & Development potential & Defination & Example \\
\hline 1. & Totipotent & These cells have the ability to form an entire organism & $\begin{array}{l}\text { Zygote up to the eight cell stage of } \\
\text { the morula }\end{array}$ \\
\hline 2. & Pluripotent & $\begin{array}{l}\text { These cells can give rise to any cell type found in the } \\
\text { primary germinal layer of the embryo, i.e. ectoderm, } \\
\text { endoderm and mesoderm }\end{array}$ & Embryonic stem cells \\
\hline 3. & Multipotent & $\begin{array}{l}\text { These cells can give rise to all cell types within } \\
\text { a particular lineage }\end{array}$ & Mesenchymal stem cells from adults \\
\hline 4. & Oligopotent & These cells have limited differentiation potential & $\begin{array}{l}\text { Stem cells from adult lymphoid/ } \\
\text { myeloid organs }\end{array}$ \\
\hline 5. & Unipotent & $\begin{array}{l}\text { These cells can differentiate into only a particular } \\
\text { defined lineage }\end{array}$ & Cells from liver, lung, etc. \\
\hline
\end{tabular}




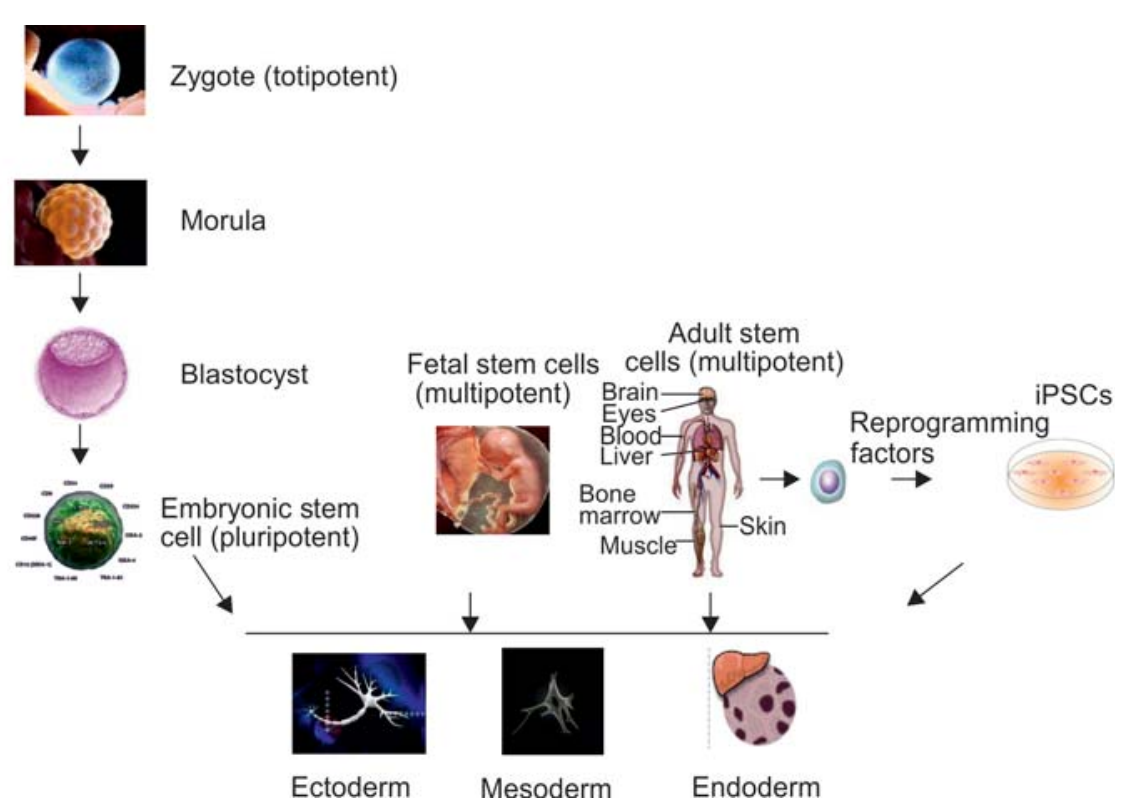

Fig. 2: Different sources of stem cells and their differentiation potential

demonstrated that UCE is an important source of the human primary keratinocytes. In UCB, we can find two different types of SCs, i.e. hematopoietic (UC-HS) and mesenchymal (UC-MS). UC-MS can produce cytokines which facilitate grafting in the donor, in vitro SC survival and it is more efficient than BM MSC graft.

\section{DISCOVERY AND CHARACTERIZATION OF STEM CELLS}

Mesenchymal stem cells were discovered as early as in 1960s when AJ Freidenstein observed that the bone marrow is a source of stem cells for mesenchymal tissues in postnatal life. ${ }^{19}$ Prior to this several other scientists have achieved important milestones in stem research and contributed to our current understanding of this area. It was Alexander A Maximow who first introduced the idea of stem cells and their ability to differentiate into other cell types in the context of hematopoiesis in his proposal of the unitarian theory of hematopoiesis in 1906. ${ }^{20}$ Two other scientists, Ernest A McCulloch and James E Till were among the first to demonstrate the clonal nature of bone marrow cell injection into irradiated mice, during which the noticed spleen of these mice developed lumps or 'spleen colonies' in proportion to the number of marrow cells injected. ${ }^{21}$ Thus, though scientists knew that adult bone marrow contained cells that could make all of the blood cell types as early as in 1960s, but it was not until 1988 that those stem cells were isolated as pure cellular population. The application took so long because the techniques for identifying stem cells have only recently been developed. Partly, this is because adult stem cells are inconspicuous in shape, size and function. They also tend to hide deep in tissues and are present only in very low numbers, which makes their identification and isolation very elusive. MSCs express a number of markers phenotypically. However, none of them are specific for these cell. According to the International Society for Cellular Therapy, human MSCs under standard culture conditions must satisfy at least three criteria: (1) they must be plasticadherent; (2) they must express C105, CD73, and CD19 and HLA-DR surface molecules; (3) they must be capable of differentiating into osteblasts, adipocytes and chondroblasts. ${ }^{22}$ However, this set of criteria is not definitive as the expression of cell surface markers can be influenced by extrinsic factors, such as those secreted by accessory cells during initial passages and it is important to note that in vitro expression of cell surface markers may not correlate with in vivo expression. Other markers that are generally accepted include CD44, CD71, Stro-1 and adhesion molecules, such as CD 106, CD166, and CD29. ${ }^{23}$ So in a broad way, the following aspects may be considered while isolating and characterizing the stem cells:

- Growing and subculturing the stem cells for many months and determining whether the cells can be regrown or subcultured, after freezing, thawing and replating. This ensures that the cells are capable of longterm growth and self-renewal. One can inspect the cultures through a microscope to see that the cells look healthy and remain undifferentiated.

- The presence of transcription factors that are typically produced by undifferentiated cells. Two of the most important transcription factors are Nanog and Oct4. ${ }^{24}$ Transcription factors help turn genes on and off at the right time, which is an important part of the processes of cell differentiation and embryonic development. In this case, both Oct 4 and Nanog are associated with 
maintaining the stem cells in an undifferentiated state, capable of self-renewal.

- Expression of particular cell surface markers that are typically produced by undifferentiated cells. The most commonly used technique in these days is flow cytometry. For identifying embryonic stem cells, the common surface markers are-TRA-1-60, TRA-1-81, SSEA-4, SSEA-3. In order to isolate hematopoietic stem cells, the commonly expressed surface markers are CD34, CD-45 and CD14 while CD-90, CD-105 and CD-71 are generally expressed by the mesenchymal stem cells. ${ }^{25}$

Pluripotency of the stem cells can be tested by (1) allowing the cells to differentiate spontaneously in cell culture, (2) manipulating the cells so they will differentiate to form cells characteristic of the three germ layers viz ectoderm, mesoderm and endoderm or (3) injecting the cells into immunocompromised mice to test for the formation of a benign tumor called a teratoma. Since the mouse's immune system is suppressed, the injected human stem cells are not rejected by the mouse immune system. It is possible to observe the growth and differentiation of human stem cells. Teratomas typically contain a mixture of many differentiated or partly differentiated cell types - an indication that the embryonic stem cell is capable of differentiating into multiple cell types. ${ }^{26}$

\section{STEM CELLS, THEIR NICHE AND DIFFERENTIATION}

In adults, stem cells reside in a physiologically limited and specialized microenvironment or, niche that supports stem cells but varies in nature and location depending on the tissue type. ${ }^{27}$ The niche is a physical anchoring site for stem cells, and adhesion molecules are involved in the interaction between stem cells and the niche and between stem cells and the extracellular matrix. The niche generates extrinsic factors that control stem cell number, proliferation and fate determination. The stem cells become activated to divide and proliferate. Stem cell proliferation depends on the dynamic niche signaling. Maintaining a balance between the proliferation signal and antiproliferation signal is the key to homeostatic regulation of stem cells. This allows the stem cells to undergo self-renewal while supporting ongoing tissue regeneration. ${ }^{28}$ Many developmental regulatory signal molecules, including Hedgehog, Wnt, bone morphogenetic proteins (BMP), fibroblast growth factors, and notch, have been shown to play roles in controlling stem cell selfrenewal and in regulating lineage fate in different systems. Any genetic mutation that leads stem cells to become independent of growth signals or to resist antigrowth signals will cause the stem cells to undergo uncontrolled proliferation and possible tumorigenesis. The differentiation of stem cells has been reported to be induced by a number of growth factors, cytokines, chemicals and transcription factors. The addition of growth factors, like basic fibroblast growth factor, bone morphogenic protein and epidermal growth factor, has been reported to direct the stem cells toward ectodermal and mesodermal lineage. ${ }^{29}$ Interleukin 3 induces the formation of macrophages, mast cells or neutrophils. ${ }^{30}$ Dexamethasone, osteopontin and ascorbate trigger osteogenic differentiation while TGF beta family oxytocin, erythropoietin, 5-azacytidin and DMSO have been implicated to direct the stem cell fate to cardiomyocyte. ${ }^{31}$ All these agents modulate the stem cell fate by up-regulation or down-regulation of one or more cell signaling pathways. Recently, it was reported that manipulating the membrane potential can also influence the cell fate and differentiation. ${ }^{32}$ Mechanical factors have been reported to be used to control the fate of stem cells and direct differentiation toward the desired cell type. ${ }^{33}$

\section{POTENTIAL APPLICATIONS COME WITH A WARNING}

The multilineage differentiation potential of stem cells is beneficial but also a challenge as differentiation at the wrong time, place or to an undesired cell type may lead to a harmful pathophysiological condition. Stem cells have evolved elaborate circuitry that helps them to respond to differentiation cues only in an appropriate biological context in order to avoid any maladaptive responses. A complex loop of genetic determinants and signaling factors are involved in maintaining the delicate balance between stem cell self-renewal and differentiation. Both embryonic and adult stem cells are potential resource for investigating early developmental processes as well as assessing the therapeutic potential of these cells in numerous disease models. The most widespread application of human bone marrow stem cells is the replacement therapies - to replace diseased or degenerating tissues or to replace cell population, such as

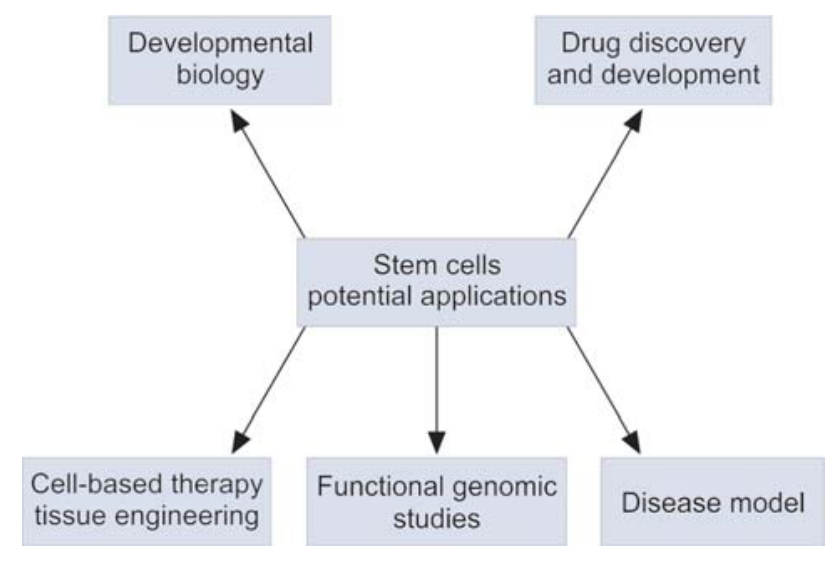


those of the hematopoietic system. It is important to understand the entire paradigm that regulates their selfrenewal and differentiation since this can open up avenues to new stem cell based therapies for developmental defects and disease. Stem cell therapy has been found useful to repair damaged spinal cords; ${ }^{34}$ cure Crohn's, ${ }^{35}$ Alzheimer's and Parkinson's disease, ${ }^{36}$ regrow arteries around a blockage, ${ }^{37}$ regrow limbs, ${ }^{38}$ replace failed kidney, ${ }^{39}$ and heart, ${ }^{40}$ cure diabetes by replacing nonfunctional cells in the pancreas, ${ }^{41}$ restore vision, ${ }^{42}$ and hearing, ${ }^{43}$ treat leukemia and lymphoma that are nonresponsive to normal therapy ${ }^{44}$ and treat brain cancer. ${ }^{45}$ All these potential applications exploit the ability of selective differentiation of stem cells under predefined culture condition. Human iPS cell-derived cardiomyocytes can also be valuable as a test system for evaluating the toxicity and efficacy of new medicines or chemicals. ${ }^{46}$ The wide varieties of cell type and tissue that may develop from stem cells represent a biological system that mimics many of the complex interactions of the cells and tissues of the body, and hence provides an attractive and valuable screening tool. This type of assay could have wide applications in the pharmaceutical, chemical, cosmetics, agrochemical industries and revolutionize traditional chemical medicine. Stem cell research may help to clarify the role genes play in human development and the role of genetic mutations and their effect on normal processes. They can be used to study how infectious agents invade and attack human cells to investigate the genetic and environmental factors that are involved in cancer and other diseases, and to decipher the process of aging. Embryonic stem cells which can continue to divide for long periods of time and produce a variety of cell types could provide a valuable source of human cells for testing drugs or measuring the effects of toxins on normal tissues without risking the health of a single human volunteer. In the future, thousands of compounds could be quickly tested on a wide assortment of cell types derived from stem cells, making drug discovery more efficient and cost effective. The induced pluripotent stem cells could be particularly useful for testing drugs for disorders that are of genetic origin. For example, it is difficult to study the progression of Alzheimer's and Parkinson's diseases in the brains of live patients, but by using the cells of an Alzheimer's patient to create stem cell lines, scientists could trace the development of the disease in a culture dish and test drugs that regenerate lost nerve cells with no danger to the patient. Stem cells may also help to evaluate the effects of toxic substances in drugs, food and the environment. ${ }^{47}$ The plethora of medical achievements stem cells could offer seems to be expanding at an incredible pace. The role of stem cells in medicine is already very real, but there is a danger of exaggerating the promise of this new medical development. It is important to understand the mechanisms regulating quiescent and differentiated state of the stem cell. This knowledge is essential to unwind the complex web of switches that push a naïve cell to more specialized state. The basic research needed to develop viable therapeutic options is a lengthy process that may extend over many years and decades. Even after science has moved from basic research to developing medical applications, it will still take a long time to thoroughly test those applications and demonstrate that they are safe to be prescribed for patients.

\section{REFERENCES}

1. Langer R, Vacanti JP. Tissue engineering. Science 1993;260: 920-26.

2. Kim PG, Daley GQ. Application of induced pluripotent stem cells to hematologio disease. Cytotherapy 2009;11:980-89.

3. Lyssiotis CA, Lairson LL, Boitano AE, et al. Chemical control of stem cell fate and developmental potential. Angew Chem Int Ed 2011;50:200-42.

4. Morrison SJ, Kimble J. Asymmetric and symmetric stem-cell divisions in development and cancer. Nature 2006;441: 1068-74.

5. Blau HM. A twist of fate. Nature 2002;419-37.

6. Takahashi K, Yamanaka S. Induction of pluripotent stem cells from mouse embryonic and adult fibroblast cultures by defined factors. Cell 2006;126:663-76.

7. Fortier LA. Stem cells: Classifications, controversies and clinical applications. Vet Surg 2005;34:415-23.

8. Hayflick L, Moorhead PS. The serial cultivation of human diploid cell strains. Exp Cell Res 1961;25:585-621.

9. Gallacher L, Murdoch B, Wu D, Karanu F, Fellows F, Bhatia M. Identification of novel circulating human embryonic blood stem cells. Blood 2000;96:1740-47.

10. Fortier LA, Nixon AJ, Williams J, Cable CS. Isolation and chondrocytic differentiation of equine bone marrow-derived mesenchymal stem cells. Am J Vet Res 1998;59:1182-87.

11. Deasy BM, Li Y, Huard J. Tissue engineering with musclederived stem cells. Curr Opin Biotechnol 2004;15:419-23.

12. De Bari C, Dell'Accio F, Tylzanowski P, Luyten FP. Multipotent mesenchymal stem cells from adult human synovial membrane. Arthritis Rheum 2001;44:1928-42.

13. Blanpain C, Lowry WE, Geoghegan A, Polak L, Fuchs E. Selfrenewal, multipotency, and the existence of two cell populations within an epithelial stem cell niche. Cell 2004;118:635-48.

14. Miura M, Gronthos S, Zhao M, et al. SHED: Stem cells from human exfoliated deciduous teeth. Proc Natl Acad Sci USA 2003;100:5807-12.

15. McKay RD. Stem cell biology and neurodegenerative disease. Philos Trans R Soc Lond B Biol Sci 2004;359:851-56.

16. Tan SC, Pan WX, Ma G, Cai N, Leong KW, Liao K. Viscoelastic behaviour of human mesenchymal stem cells. BMC Cell Biol 2008;9:40.

17. Meirelles Lda S, Nardi NB. Methodology, biology and clinical applications of mesenchymal stem cells. Front Biosci 2009;14: 4281-98.

18. Ruhil S, Kumar V, Rathee P. Umbilical cord stem cell: An overview. Curr Pharm Biotechnol 2009;10:327-34. 
19. Afanasyev BV, Elstner EE, Zander AR. AJ friedenstein, founder of the mesenchymal stem cell concept. Cellular therapy and Transplantation 2009;1(3);35-38.

20. Maximow AA. About experimental generation of bone marrow tissue. Anatomical Gazette 1906;28:24-38.

21. Siminovitch L, McCulloch EA, Till JE. Cytological demonstration of the clonal nature of spleen colonies derived from transplanted mouse marrow cells. Nature 2009;197(3):31-34.

22. Dominici M, Le Blanc K, Mueller I, et al. Minimal criteria for definingmultipotentmesenchymal stromal cells: The international society for cellular therapy position statement. Cytotherapy 2006;8(4)315-17.

23. Chamberlain G, Fox J, Ashton B, Middleton J. Concise review: Mesenchymal stem cells. Their phenotype, differentiation capacity, immunological features, and potential for homing. Stem Cells 2007;25(11):2739-49.

24. Chambers I, Colby D, Robertson M, et al. Functional expression cloning of Nanog, a pluripotency sustaining factor in embryonic stem cells. Cell 2003;113:643-55

25. Raman JD, Mongan NP, Liu L, et al. Decreased expression of the human stem cell marker, Rex-1 (zfp-42), in renal cell carcinoma. Carcinogenesis 2006;27:499-507.

26. Pittenger MF, Mackay AM, Beck SC, et al. Multilineage potential of human mesenchymal stem cells. Science 1999;284: 143-47.

27. Burdick JA, Watt FM. High-throughput stem-cell niches. Nature Methods 2011;11:915-16.

28. Lodi D, Iannitti T, Palmieri B. Stem cells in clinical practice: Applications and warnings. J Exp Clin Cancer Res 2011;30:9.

29. Hollnagel A, Oehlmann V, Heymer J, Ruther U, Nordheim A. Id genes are direct targets of bone morphogenetic protein induction in embryonic stem cells. J Biol Chem 1999;274: 19838-45.

30. Wiles MV, Keller G. Multiple hematopoietic lineages develop from embryonic stem (ES) cells in culture. Development 1991; 111:259-67.

31. Kim DH, Yoo KH, Choi KS, et al. Gene expression profile of cytokine and growth factor during differentiation of bone marrow-derived mesenchymal stem cell. Cytokine 2005;31: 119-26.

32. Sundelacruz S, Levin M, Kaplan DL. Membrane potential controls adipogenic and osteogenic differentiation of mesenchymal stem cells. PLoS One 2008;3:e3737.

33. Kilian KA, Bugarija B, Lahn BT, Mrksich M. Geometric cues for directing the differentiation of mesenchymal stem cells. Proc Natl Acad Sci USA 2010;107:4872-77.

34. Salehi M, Pasbakhsh P, Soleimani M, et al. Repair of spinal cord injury by cotransplantation of embryonic stem cell-derived motor neuron and olfactory ensheathing cell. Iran Biomed J 2009;13:125-35.
35. Singh UP, Singh NP, Singh B, et al. Stem cells as potential therapeutic targets for inflammatory bowel disease. Front Biosci 2010;2:993-1008.

36. Lunn JS, Sakowski SA, Hur J, Feldman EL. Stem cell technology for neurodegenerative diseases. Ann Neurol 2011;70:353-61.

37. Glaser DE, Gower RM, Lauer NE, et al. Functional characterization of embryonic stem cell derived endothelial cells. J Vasc Res 2011;48:415-28.

38. Tamura K, Ohgo S, Yokoyama H. Limb blastema cell: A stem cell for morphological regeneration. Dev Growth Differ 2010;52: 89-99.

39. Hendry C, Rumballe B, Moritz K, Little MH. Defining and redefining the nephron progenitor population. Pediatr Nephrol 2011;26:1395-1406.

40. Otto WR, Wright NA. Mesenchymal stem cells: From experiment to clinic. Fibrogenesis Tissue Repair 2011;4:20.

41. LoCascio SA, Spinelli J, Kurtz J. Hematopoietic stem cell transplantation for the treatment of autoimmunity in type 1 diabetes. Curr Stem Cell Res Ther 2011;6:29-37.

42. Singh MS, MacLaren RE. Stem cells as a therapeutic tool for the blind: Biology and future prospects. Proc Biol Sci 2011;278: 3009-16.

43. Huisman MA, Rivolta MN. Neural crest stem cells and their potential application in a therapy for deafness. Front Biosci 2012;4:121-32.

44. Watanabe J, Kondo H, Hatake K. Autologous stem cell transplantations for recurrent adult $\mathrm{T}$ cell leukaemia/lymphoma using highly purified CD34p cells derived from cryopreserved peripheral blood stem cells. Leuk Lymphoma 2001;42: 1115-17.

45. Werbowetski-Ogilvie TE, Coudiere Morrison L, FiebigComyn A, Bhatia M. In vivo generation of neural tumors from neoplastic pluripotent stem cells models early human pediatric brain tumor formation. Stem Cells 2012;30:392-404.

46. Guo L, Abrams RM, Babiarz JE, et al. Estimating the risk of drug-induced proarrhythmia using human induced pluripotent stem cell-derived cardiomyocytes. Toxicol Sci 2011;123: 281-89.

47. Jiang X, Lu S. Research advances in induced pluripotent stem cells. Zhongguo Yi Xue Ke Xue Yuan Xue Bao 2011;33: 456-61.

\section{ABOUT THE AUTHOR}

\section{Shamoli Bhattacharyya}

Assistant Professor, Department of Biophysics, Postgraduate Institute of Medical Education and Research, Chandigarh, India Phone: 9876186816, e-mail: shalmoli2007@yahoo.co.in 